\title{
Myxedema Associated with Cardiac Tamponade
}

\author{
Chih-Ta LIN, ${ }^{1} \mathrm{MD}$, Chih-Jen LIU, ${ }^{1} \mathrm{MD}$, Tin-Kwang LIN, ${ }^{1} \mathrm{MD}$, \\ Chih-Wei CHEN, ${ }^{1} \mathrm{MD}$, Bin-Chen CHEN, ${ }^{1} \mathrm{MD}$, and Chih-Lon LIN, ${ }^{1} \mathrm{MD}$
}

\begin{abstract}
SUMMARY
Pericardial effusion is frequently found in patients diagnosed with hypothyroidism, yet it is rarely associated with cardiac tamponade. This report presents an atypical case concerning a 60-year-old Taiwanese female, with a history of thyroidectomy surgery due to thyroid cancer, who was later diagnosed with myxedema and cardiac tamponade. Treatment included an immediate pericardiocentesis followed by thyroxine hormone replacement therapy. Postoperative and recovery phases progressed favorably, and the patient's prognosis is good. (Jpn Heart J 2003; 44: 447-450)
\end{abstract}

Key words: Pericardial effusion, Myxedema, Cardiac tamponade

GORDON (1929) was the first to document hypothyroidism coupled with pericardial effusion. Reports based on patients left untreated for hypothyroidism indicate pericardial effusion in $30-78 \%$ of those surveyed, ${ }^{1-2)}$ while Kabadi, et al reported figures that are considerably lower (3\%-5\%) in patients promptly diagnosed during the early stages of the disease. ${ }^{3)}$ Hypothyroidism complicated by cardiac tamponade is rarely referenced in the medical literature. This report presents the atypical case of a female patient diagnosed with myxedema and cardiac tamponade, which subsided following immediate pericardiocentesis and thyroxine hormone replacement. The mechanisms for the formation of the pericardial effusion and biochemical changes in hypothyroidism patients are also discussed.

\section{CASE REPORT}

A 60-year-old Taiwanese female experiencing shortness of breath and chest discomfort was admitted to hospital on November 30, 2001. Patient history included thyroid cancer and a total thyroidectomy. Thyroxine had been recommended following surgery, however, the patient chose not to take the medication as prescribed and within two months her condition began to deteriorate to general

From the ${ }^{1}$ Cardiovascular Division, Department of Internal Medicine, Buddhist Dalin Tzu Chi General Hospital, Chia-Yi, Taiwan.

Address for correspondence: Tin-Kwang Lin, MD, Buddhist Dalin Tzu General Hospital, No.2, Min-Sheng Road, Dalin Town, Chia-Yi, Taiwan.

Received for publication June 20, 2002.

Revised and accepted September 20, 2002. 
malaise, lethargy, slow speech, edema of the face and extremities, and progressive weight gain. Vital signs on admission were recorded (temperature, $37 \mathrm{C}^{\circ}$; blood pressure, 101/73 $\mathrm{mmHg}$; heart rate, regular at 76 beats/min). Physical examination found facial edema, coarse hair, dry skin, an engorged jugular vein, a distant heart sound, nonpitting edema of the extremities, and a nonpalpable thyroid. Chest roentgenogram showed obvious globular enlargement of the cardiac silhouette (Figure 1A). An electrocardiogram revealed low $\mathrm{P}$ wave and QRS complexes. An echocardiogram demonstrated normal heart size but with massive pericardial effusion and signs of early systolic RA and early diastolic RV collapse (Figure 2). Cardiac tamponade was diagnosed and pericardiocentesis was
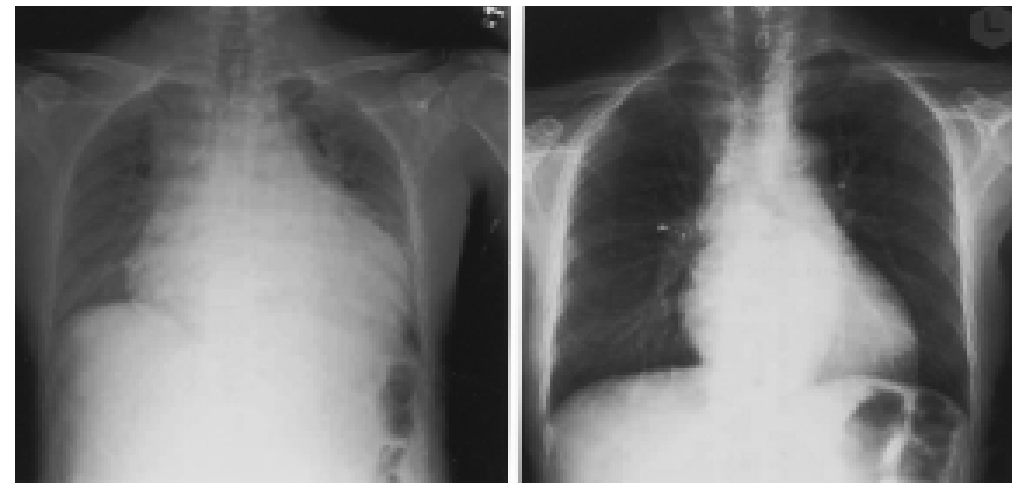

A

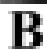

Figure 1. A: Chest roentgenogram made upon admission shows obvious globular cardiac enlargement suggesting massive pericardial effusion. B: Chest roentgenogram four months following thyroxine treatment shows normal cardiac silhouette.

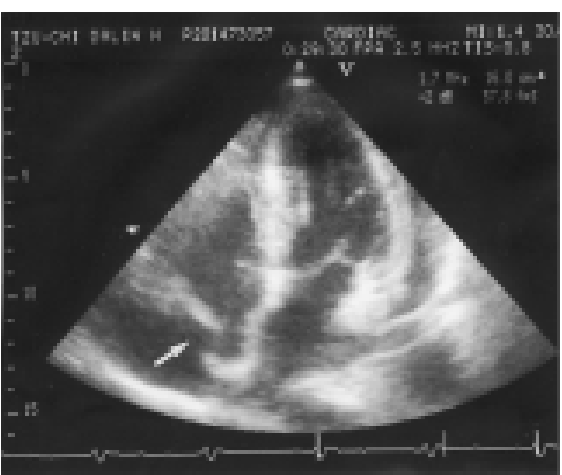

A

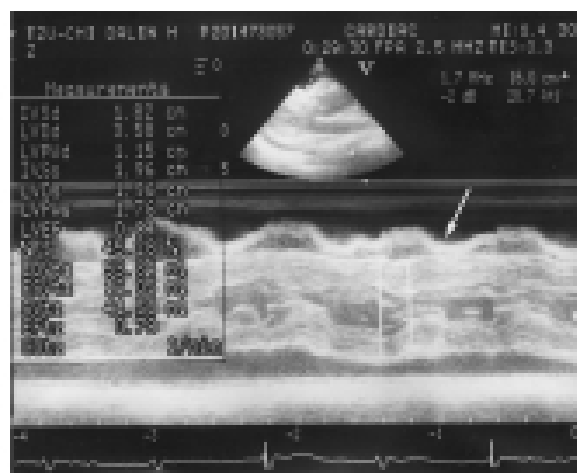

B

Figure 2. A: Four chamber view with two-dimensional echocardiography shows collapse of the right atrial free wall (arrow). B: M-mode echocardiography with parasternal approach shows diastolic collapse of the right ventricle free wall (arrow). 
performed immediately. Seven hundred and twenty $\mathrm{mL}$ of a clear yellow fluid with a protein content of $5.3 \mathrm{gm} / \mathrm{dL}$ was drained. After the procedure, her heart rate decreased to 60 beats/min and blood pressure increased to $130 / 90 \mathrm{mmHg}$. A blood chemistry profile gave the following results: aspartate aminotransferase (AST) 206 IU/L, alanine aminotransferase (ALT) 129 IU/L, total cholesterol 287 $\mathrm{mg} / \mathrm{dL}$, high-density lipoprotein cholesterol (HDL-C) $72 \mathrm{mg} / \mathrm{dL}$, triglyceride: 77 $\mathrm{mg} / \mathrm{dL}$, sodium $132 \mathrm{mEq} / \mathrm{L}$, serum thyroid-stimulating hormone (TSH): $74 \mathrm{mU} /$ $\mathrm{mL}$ (normal 0.35-5.5 mU/mL), T3: $25.26 \mathrm{ng} / \mathrm{dL}$ (normal 90-190 ng/dL), and T4 $0.66 \mu \mathrm{g} / \mathrm{dL}$ (normal 5-12 $\mu \mathrm{g} / \mathrm{dL}$ ).

Throughout the whole course, the patient had no viral infection or fever history. She also had a normal white blood cell count and normal C-reactive protein $(\mathrm{CRP}<0.4 \mathrm{mg} / \mathrm{dL})$. The pericardial effusion was sent for bacterial and tuberculosis cultures and was negative. All other causes of cardiac tamponade except hypothyroidism were ruled out. After hypothyroidism was diagnosed, thyroxine therapy $(0.1 \mathrm{mg}$ per day) was prescribed. The facial and extremity edema subsided and her body weight decreased gradually. Four months later, a second profile gave the following results: AST 24 IU/L, ALT: 18 IU/L, total cholesterol 198 $\mathrm{mg} / \mathrm{dL}, \mathrm{HDL}-\mathrm{C}$ : $63 \mathrm{mg} / \mathrm{dL}$, triglyceride $82 \mathrm{mg} / \mathrm{dL}$, TSH $3.3 \mathrm{mU} / \mathrm{mL}$, T3 $128 \mathrm{ng} /$ $\mathrm{dL}$, and T4 $10 \mu \mathrm{g} / \mathrm{dL}$. A chest $\mathrm{x}$-ray taken four months after discharge showed that the cardiac silhouette had returned to normal size (Figure 1B). The patient is currently receiving follow-up care through the outpatient department, and her prognosis is good.

\section{DISCUSSION}

The accumulation of proteinaceous fluid in vital body cavities of persons diagnosed with hypothyroidism is widely documented, the most common sites being the pericardial, pleural, and peritoneal cavities. ${ }^{4)}$ Pericardial effusion has been reported frequently in adults diagnosed with hypothyroidism. However, recent research suggests that early diagnosis of the disease precludes the occurrence of pericardial effusion. General consensus concludes that this complication may be attributed to increased capillary permeability and subsequent leakage of fluid high in protein into the interstitial space, impaired lymphatic drainage, and a greater retention of salt and water. ${ }^{5,6}$ However, the occurrence of cardiac tamponade in patients diagnosed with hypothyroidism is rare, possibly due to slow accumulation of fluid and pericardial distensibility, which contributes to significant fluid collection without hemodynamic compromise. ${ }^{7)}$ The extravascular accumulation of albumin can be detected after 2-3 months of hypothyroidism. The total protein content is high in pericardial effusion. Pericardiocentesis is the most effective treatment for immediate relief of tamponade. But unless tampon- 
ade is present, pericardiocentesis may not be necessary since thyroid replacement therapy can correct the effusion.

Patients with cardiac tamponade usually display tachycardia, but in this case, the initial heart rate was only 76 beats $/ \mathrm{min}$, which decreased to 60 beats/min after pericardiocentesis. This is because hypothyroid patients have baseline bradycardia and their heart rate does not elevate significantly even when cardiac tamponade is present.

It has been suggested that patients with hypothyroidism are at risk of developing atherosclerosis since this disease is associated with hyperlipidemia. All cholesterol subfractions are increased, including LDL, VLDL, and HDL cholesterol, but all lipid abnormalities can be readily reversed with thyroxine replacement. The present case exhibited elevated total cholesterol and HDL-C levels, which returned to normal ranges with simple thyroxine replacement therapy. Elevated aspartate aminotransferase and alanine aminotransferase levels were also noted, but the actual reason is not clear. The patient had no history of liver disease, although initial AST and ALT test results were elevated, however, they also returned to normal ranges after thyroxine treatment. These changes are significant and have seldom appeared in earlier research.

In conclusion, myxedema with cardiac tamponade is a rare condition. Pericardial effusion, lipid metabolic abnormality and abnormal liver function can be easily reversed with thyroid replacement. Pericardiocentesis is necessary only when tamponade develops.

\section{REFERENCES}

1. Hardisty CA, Naik DR, Munro DS. Pericardial effusion in hypothyroidism. Clin Endocrinol 1980; 13(4): 34954.

2. Kurtzman RS, Otto D, Chepy JJ. Myxoedema heart disease. Radiology 1965; 84: 624.

3. Kabadi, Udaya M, Kumar SP. Pericardial effusion in primary hypothyroidism. Am Heart J 1990; 120: 1393-5.

4. Sachdev Y, Hall R. Effusions into body cavities in hypothyroidism. Lancet 1975; i: 564-6.

5. Hollenberg M, Dougherty J. Lymph flow and I-131 albumin resorption from pericardial effusion in man. Am J Cardiol 1969; 24: 514.

6. Parving HH, Hansen JM, Nielsen SL, et al, Mechanism of edema formation in myxedema-increased protein extravasation and relatively slow lymphatic drainage. N Engl J Med 1979; 301: 460.

7. Smolar EN, Rubin JE, Avramides A, et al, Cardiac tamponade in primary myxedema and review of the literature. Am J Med Sci 1976; 272: 345-52. 